\title{
VALUe Chain MANAgement in MARINe Fisheries: A CASE STUdy OF ANDHRA PRADESH
}

\author{
V V Devi Prasad Kotni \\ Department of Management Studies \\ Gayatri Vidya Parishad College for Degree and PG Courses (A), Visakhapatnam, India
}

\begin{abstract}
The value chain management assists the stakeholders to perform value added operations, add value to the product and have better returns from the market. This paper attempts at value chain management practices adopted by the players of the marine fisheries chain in Andhra Pradesh and how much value they are adding to the final fish product. The study has been structured to capture the essence of the processes and flow of the value chain in Marine fisheries. The study is designed in such a way that it addresses various research questions like the basic value chain structure, value added operations in fisheries value chain, cost of each operation along with price increase and finally the final price of the product. In this paper, in order to suggest the suitable value chain, a thorough market research was conducted to study the Value Chain Management practices employed by Fishermen, Middlemen, Retailers, Head loaders and Cycle carrier with respect to fresh fish in the coastal area of the State of Andhra Pradesh, India.
\end{abstract}

\section{INTRODUCTION}

The Value Chain, also known as Value Chain Analysis, is a concept from business management that was first described and popularized by Michael Porter in his 1985 best-seller book, Competitive Advantage: Creating and Sustaining Superior Performance. Michael Porter coined the term value system which means a larger interconnected value chains. Porter (1985) described the value system right from the supplier to the customer. The value system integrates the supplier of the firm (and their suppliers all the way back), the production centre or the firm itself, the distribution channels and the buyers of the firm. Another value chain thinker Lynch (2003) described value chain as a series of activities which includes purchasing, manufacturing, distributing and marketing of company's products.

If an attempt is made to understand the insights of value chain concept, some of the individuals and institutions have given their understandings. Porter (1990) in his book opined that the Value Chain framework is an interdependent system or network of activities, connected by linkages. Pathania-Jain (2001) proposed that the system should be managed carefully so that the linkages can be vital source of competitive advantage. Lynch (2003) discussed about value chain analysis and said that it entails the linkage of two areas which are the value of organizational activities with its main functional parts and the second the assessment of the contribution of each part in the overall added value.

World Business Council for Sustainable Development (WBCSD) defined as value chain refers to full life cycle of a product or process which includes sourcing of raw material, production, consumption and finally disposal of the product. Sustainability, UNEP and UNGC (2008) referred value chain as it encompasses thinking about the value created by the chain, particularly for end-use customers. 
Kaplinsky, R. and M. Morris (2001) in their book titled "A Handbook for Value Chain Research" which was prepared for the International Development Research Centre (IDRC) described value chain as the full range of activities, which are required to bring a product or service from conception, through the intermediary phases of production (involving a combination of physical transformation and the input of various producer services), delivery to final consumers, and final disposal after use.

Porter (1985) has given a definition of value chain in his book "Competitive Advantage" as the basic tool for diagnosing competitive advantage and finding ways to enhance it is the value chain, which divides a firm into the discrete activities it performs in designing, producing, marketing, and distributing its products. While Porter in 1990 said that value chain concept decomposes all activities of one firm into parts and analyses each individual activity and their interdependence".

Brown (1997) considers, as a tool the value chain divides a business into strategically relevant activities. Through the company is able to identify the sources of competitive advantage and perform these activities more cheaply or better than its competitors. Walters and Lancaster (2000) has offered value chain definition as a business system which creates end user satisfaction (i.e. value) and realizes the objectives of other member stakeholders.

Keeping in view, the advantages of value chain management, in this paper, an attempt has been made to evaluate the Value Chain Management practices employed by fishermen, Middlemen, Retailers, Head loaders and Cycle career with respect to fresh fish and dry fish in the State of Andhra Pradesh, India.

\section{OBJECTIVES OF THE STUDY}

The study entitled "Value Chain Management in Marine Fisheries: A Case Study of Andhra Pradesh" has been structured to capture the essence of the processes and flow of the value chain in Marine fisheries. The study is designed in such a way that it addresses various research questions like the basic value chain structure, value added operations in fisheries value chain, cost of each operation along with price increase and finally the final price of the product. The specific objectives of the study are:

$>$ To identify the different Value Chain Actors in marine fisheries sector.

$>$ To make comprehensive study on various value chain actors, their role in value chain management of fisheries in relation to various markets.

$>$ To assess various operational costs involved at each stage of fish catch to processing.

$>$ To suggest the suitable value chains to the key stakeholders of the sector.

\section{REVIEW OF LITERATURE}

While conducting desk research of previous studies, it was found that there were some researchers who did value chain analysis earlier in some sectors, industries and even in some management functions.

$>$ Ching Chyi Lee and Jie Yang (2000) conducted value chain research in knowledge sector and proposed a knowledge value chain model.

$>$ Vorster (2001) organised value chain research in mines sector and proposed mining value chain.

$>$ Vander Merwe and Cronje (2004) organised value chain management research in education sector and proposed education value chain.

$>$ Gabriel (2006) has done value chain research in service sector and proposed value chain framework for customised services.

$>$ Rijean Landry et al. (2006) has done research in health sector and designed value chain model for health organisations. 
Ilyas et al. (2008) conducted research in BPO sector and determined outsourcing of value chain activities effectively.

$>$ Wang Aimin and Li Sunxi (2011) proposed value chain management in CRM function.

$>$ Seyed Mohammad Adeli et al. (2012) proposed charity value chain.

\section{Methodology OF THE STUdY}

The study is descriptive research, empirical evidence is provided and the variables used are quantitative in nature. The study has adopted some tools and techniques for collection of information on various aspects. Some of them are detailed below.

$>$ Secondary Research

$>$ Primary survey through structured questionnaires administered to key stakeholders

\subsection{Secondary Research}

Under secondary research, a thorough desk review was done to develop insights into the key areas that needs to be focused during the primary research and discussions were held with various knowledgeable persons in the Marine Fisheries sector to collect information related to the issue on hand. Collection of Secondary Data from various sources i.e., Census of India, Statistics and Planning Board, CMFRI, CIFT, CIFRI, NIO, MPEDA.

\subsection{Primary Research}

The survey was conducted using different questionnaires for different players of the fishery value chain from nine Coastal Districts of Andhra Pradesh covering $974 \mathrm{Kms}$ of coastline from Srikakulam to Nellore (From Major Fish Landing Centres) to collect first-hand information on the specific parameters developed on the basis of objectives of the study. Separate questionnaires were administered to each key stakeholder of the study i.e. fishermen, middlemen, head loader, cycle carrier and retailer.

\subsection{Study area}

The study entitled "A Study on Value Chain Management in Marine Fisheries: A Special Reference to Andhra Pradesh" was conducted according to the preferential sampling of the researcher. The selection of the study area is on the basis of importance of the Marine Fish Landing Centers among the 9 Coastal Districts of Andhra Pradesh covering $974 \mathrm{kms}$ of coastline from Srikakulam to Nellore, the number of fisherman at the landing center, the volume of trade generated, etc. The major regional market for fishery was covered during the study to develop a good understanding of the marine fishery value chain.

\subsection{Sampling}

The sample frame was designed in such a way that all the key stakeholders are covered i.e. fishermen, middlemen, head loader, cycle carrier and retailers in the study area so that the complete picture of marine fisheries value chain can be assessed as it is presented in Table 1 in which the details about study area and sample size are mentioned with different respondents. Quota Sampling Technique was adopted to collect data from their respective coastal districts. Simple Random Technique was adopted to collect data within a district. 
Table 1. Sampling according to Study Area

\begin{tabular}{|c|c|c|c|c|c|c|c|c|c|c|}
\hline \multirow[b]{2}{*}{ Respondent } & \multicolumn{9}{|c|}{ Coastal Districts of Andhra Pradesh } & \multirow[b]{2}{*}{ TOTAL } \\
\hline & 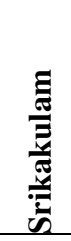 & 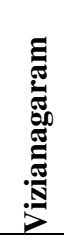 & 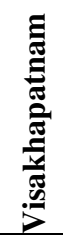 & 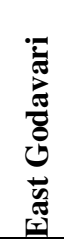 & 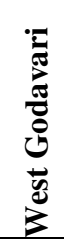 & 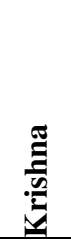 & $\stackrel{\Xi}{\Xi}$ & $\frac{\check{\Xi}}{\bar{\Xi}}$ & 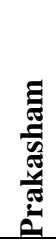 & \\
\hline Fishermen & 50 & 50 & 50 & 50 & 50 & 50 & 50 & 50 & 50 & 450 \\
\hline Middlemen & 8 & 8 & 8 & 8 & 8 & 8 & 8 & 8 & 8 & 72 \\
\hline Head loader & 10 & 10 & 10 & 10 & 10 & 10 & 10 & 10 & 10 & 90 \\
\hline Cycle Carrier & 10 & 10 & 10 & 10 & 10 & 10 & 10 & 10 & 10 & 90 \\
\hline Retailers & 20 & 20 & 20 & 20 & 20 & 20 & 20 & 20 & 20 & 180 \\
\hline Total & & & & & & & & & & 882 \\
\hline
\end{tabular}

\section{IDENTIFICATION OF VALUE CHAIN ACTORS PERFORMING VALUE CHAIN OPERATIONS}

Table 2: Value Chain actors

\begin{tabular}{|c|c|c|c|}
\hline $\begin{array}{c}\text { Value Chain } \\
\text { Actor }\end{array}$ & $\begin{array}{l}\text { Performing } \\
\text { Value Chain }\end{array}$ & $\begin{array}{c}\text { Not } \\
\text { Performing } \\
\text { Value Chain }\end{array}$ & TOTAL \\
\hline Fishermen & $\begin{array}{c}378 \\
(84.00)\end{array}$ & $\begin{array}{c}72 \\
(16.00)\end{array}$ & $\begin{array}{c}450 \\
(100.00)\end{array}$ \\
\hline Middlemen & $\begin{array}{c}54 \\
(75.00) \\
\end{array}$ & $\begin{array}{c}18 \\
(25.00) \\
\end{array}$ & $\begin{array}{c}72 \\
(100.00) \\
\end{array}$ \\
\hline Head loader & $\begin{array}{c}72 \\
(80.00)\end{array}$ & $\begin{array}{c}18 \\
(20.00)\end{array}$ & $\begin{array}{c}90 \\
(100.00)\end{array}$ \\
\hline Cycle Carrier & $\begin{array}{c}72 \\
(80.00)\end{array}$ & $\begin{array}{c}18 \\
(20.00)\end{array}$ & $\begin{array}{c}90 \\
(100.00)\end{array}$ \\
\hline Retailer & $\begin{array}{c}126 \\
(70.00)\end{array}$ & $\begin{array}{c}54 \\
(30.00)\end{array}$ & $\begin{array}{c}180 \\
(100.00)\end{array}$ \\
\hline TOTAL & $\begin{array}{c}702 \\
(80.00)\end{array}$ & $\begin{array}{c}180 \\
(20.00)\end{array}$ & $\begin{array}{c}882 \\
(100.00)\end{array}$ \\
\hline
\end{tabular}

From the sample what was obtained from the study area, the respondents (i.e. key stakeholders) were divided into two categories i.e. performing value chain and not-performing value chain. From Table 2, it can be found that there are 378 (84\%) fishermen who perform value chain operations and $72(16 \%)$ fishermen who are not performing value chain operations. Among the sample, 54 (75\%) middlemen who are performing value chain operations and $18(25 \%)$ who are not performing value chain operations. Among the head loaders, $72(80 \%)$ head loaders who are performing value chain operations and $18(20 \%)$ who are not performing value chain operations. There are $72(80 \%)$ cycle carriers who perform value chain operations and $18(20 \%)$ cycle carriers who are not performing value chain operations. Among the retailers, $126(70 \%)$ are 
performing value chain operations and $54(30 \%)$ retailers who are not performing value chain operations. Among the total sample, $702(80 \%)$ head loaders who are performing value chain operations and $180(20 \%)$ respondents who are not performing value chain operations.

\section{Calculation Of Prices of fish without Value Chain Operations}

In this section, an attempt has been made to calculate prices of fish sold out without performing Value Chain Operations. Table 3 presents cost sale price, profit and profit percentage getting by value chain actors. In order to calculate prices, the following parameters were observed as factors that influence price of fish.

1. Fisherman price at sea shore.

2. Middleman commission.

3. Transportation Charges.

4. Market fee.

5. Wholesaler margin.

6. Retailer Margin.

Table 3. Average Price of Fish without Value Chain Operations

\begin{tabular}{|c|c|c|c|c|}
\hline $\begin{array}{c}\text { Value Chain } \\
\text { Actor }\end{array}$ & $\begin{array}{c}\text { Cost of } \\
\text { Fish } \\
\text { Rs./Kg }\end{array}$ & $\begin{array}{c}\text { Sale } \\
\text { Price } \\
\text { Rs./Kg }\end{array}$ & $\begin{array}{c}\text { PROFIT } \\
\text { Rs./Kg }\end{array}$ & $\begin{array}{c}\text { \% of } \\
\text { PROFIT }\end{array}$ \\
\hline $\begin{array}{c}\text { Fishermen } \\
\text { Intermediary } \\
\text { Marketing }\end{array}$ & 35 & 42 & 7 & $17 \%$ \\
\hline $\begin{array}{c}\text { Fishermen } \\
\text { Direct Marketing } \\
\text { Consumer }\end{array}$ & 35 & 48 & 13 & $27 \%$ \\
\hline $\begin{array}{c}\text { Fishermen } \\
\text { Direct Marketing } \\
\text { Company }\end{array}$ & 35 & 60 & 25 & $42 \%$ \\
\hline Middlemen & 42 & 55 & 13 & $24 \%$ \\
\hline Head loader & 55 & 75 & 20 & $27 \%$ \\
\hline Cycle Carrier & 55 & 80 & 25 & $31 \%$ \\
\hline Retailer & 55 & 65 & 10 & $15 \%$ \\
\hline
\end{tabular}

\section{Identified General VAlue Chain Operations by FiShermen}

Table 4 presents general value chain operations adopted by fishermen for fresh fish to increase value there by price. The identified value chain operations performed by the fishermen for fresh fish are Cleaning, Washing, Separating, Grading, Weighing, Icing and Packaging. The average cost incurred by the fishermen was found to be Rs.11.83 per kg and average price increased found to be Rs.21.38 per kg. Hence there was increase of Rs.9.55/- per kg (55\%) (Price increase - cost incurred) for fresh fish. 
International Journal of Managing Value and Supply Chains (IJMVSC) Vol. 7, No. 2, June 2016

Table 4. Cost and Price addition through Value Chain for Fresh Fish

\begin{tabular}{|l|l|l|l|}
\hline Particulars of Item & $\begin{array}{l}\text { Cost } \\
\text { Rupees/Kg }\end{array}$ & $\begin{array}{l}\text { Price } \\
\text { Increase }\end{array}$ & $\begin{array}{l}\text { \% } \\
\text { Price } \\
\text { Increase }\end{array}$ \\
\hline Value Chain Operations (fresh fish) & \multicolumn{3}{l|}{} \\
\hline Cleaning & 1.50 & 3.76 & $18 \%$ \\
\hline Washing & 1.89 & 2.98 & $14 \%$ \\
\hline Separating & 0.97 & 1.89 & $9 \%$ \\
\hline Grading & 0.59 & 1.90 & $9 \%$ \\
\hline Weighing & 1.79 & 2.75 & $13 \%$ \\
\hline Icing & 2.89 & 3.98 & $19 \%$ \\
\hline Packaging & 2.20 & 4.12 & $19 \%$ \\
\hline Total & 11.83 & 21.38 & $100 \%$ \\
\hline $\begin{array}{l}\text { Value addition for Fresh Fish per Kg } \\
\text { (Price - Cost) PROFIT }\end{array}$ & 9.55 & $55 \%$ \\
\hline
\end{tabular}

\section{Fresh Fish VAlue Chain Operations by Fishermen PRACTICING INTERMEDIARY MARKETING}

Table 5. Fresh Fish Value Chain with Intermediary Marketing - Fishermen

\begin{tabular}{|l|c|c|}
\hline Particulars of Item & $\begin{array}{l}\text { Values in } \\
\text { Rs. / Kg }\end{array}$ & $\begin{array}{l}\text { Values in } \\
\%\end{array}$ \\
\hline Value Addition Operations by fisherman \\
\hline Fisherman Price at Seashore & 35.00 & $61 \%$ \\
\hline Transportation cost to Village & 0.80 & $1 \%$ \\
\hline Value Addition Process 1 - Cleaning & 3.76 & $7 \%$ \\
\hline Value Addition Process 2 -Washing & 2.98 & $5 \%$ \\
\hline Value Addition Process 3 -Separating & 1.89 & $3 \%$ \\
\hline Value Addition Process 4 -Grading & 1.90 & $3 \%$ \\
\hline Value Addition Process 5 -Weighing & 2.75 & $5 \%$ \\
\hline Value Addition Process 6 -Icing & 3.98 & $7 \%$ \\
\hline Value Addition Process 7 -Packaging & 4.12 & $7 \%$ \\
\hline Final Avg. sale price (for Middlemen / Retailer) & & 57.18 \\
\hline PROFIT addition & 22.18 & $38 \%$ \\
\hline Final Sale Price - Seashore Price ) & & \\
\hline
\end{tabular}

Table 5 shows, fishermen cost of fish Rs.35/kg, transportations charges Rs.0.80/Kg, after value addition operations like cleaning (Rs.3.76/kg), Washing (Rs.2.98/kg). Separating (Rs.1.89/kg), Grading (Rs.1.90/kg), Weighing (Rs. 2.75/kg), Icing (3.98/kg) and Packaging (4.12/kg), the value has been increased to Rs.57.18/Kg. Overall there is 38\% (Profit) price increase added to the product. 
International Journal of Managing Value and Supply Chains (IJMVSC) Vol. 7, No. 2, June 2016

\section{Fresh fish VAlue Chain Operations by fishermen PRACTICING DIRECT MARKETING}

This section concentrates on direct marketers in fishing community who practice direct marketing and involving in value chain operations. The fishermen are selling their product directly to consumer and sometimes to Exporter / Fish Companies also.

Table 6. Fresh Fish Value Chain with Direct Marketing - Fishermen

\begin{tabular}{|c|c|c|}
\hline Particulars of Item & $\begin{array}{l}\text { Values in } \\
\text { Rs. / Kg }\end{array}$ & $\begin{array}{l}\text { Values in } \\
\%\end{array}$ \\
\hline \multicolumn{3}{|l|}{ Value Addition Operations by fisherman } \\
\hline Fisherman Price at Seashore & 35.00 & $61 \%$ \\
\hline Transportation cost to Village & 0.80 & $1 \%$ \\
\hline Value Addition Process 1 - Cleaning & 3.76 & $7 \%$ \\
\hline Value Addition Process 2 -Washing & 2.98 & $5 \%$ \\
\hline Value Addition Process 3 -Separating & 1.89 & $3 \%$ \\
\hline Value Addition Process 4 -Grading & 1.90 & $3 \%$ \\
\hline Value Addition Process 5 -Weighing & 2.75 & $5 \%$ \\
\hline Value Addition Process 6 -Icing & 3.98 & $7 \%$ \\
\hline Value Addition Process 7 -Packaging & 4.12 & $7 \%$ \\
\hline Final Avg. sale price & \multicolumn{2}{|c|}{57.18} \\
\hline \multicolumn{3}{|l|}{ Fisherman Selling fish to Consumer Directly } \\
\hline Avg.Sale Price fixed by Fisherman & 57.18 & $77 \%$ \\
\hline Transportation Chagres & 4.36 & $6 \%$ \\
\hline Packing & 0.59 & $1 \%$ \\
\hline Fisherman Margin & 12.15 & $16 \%$ \\
\hline Final Avg.Price for Consumer & \multicolumn{2}{|c|}{74.28} \\
\hline 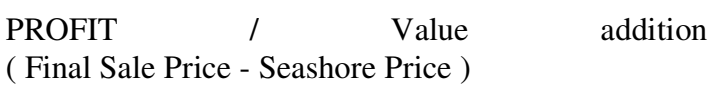 & 39.28 & $52 \%$ \\
\hline \multicolumn{3}{|l|}{ Fisherman Selling fish to Company Directly } \\
\hline Avg.Sale Price fixed by Fisherman & 57.18 & $66 \%$ \\
\hline Fisherman Margin & 30.00 & $34 \%$ \\
\hline Final Avg.Price for fish company / Exporter & \multicolumn{2}{|c|}{87.18} \\
\hline 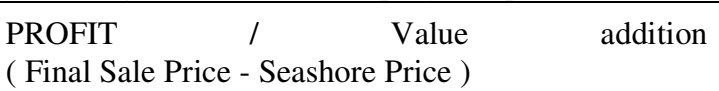 & 52.18 & $59 \%$ \\
\hline
\end{tabular}

Table 6 shows that there is more than $50 \%$ value increased to the product after performing value chain operations by fishermen. The fishermen selling fish directly to consumer are getting $52 \%$ more returns on their fish catch whereas the fishermen selling fish directly to company/ exporter are getting 59\% more returns on their fish. Here it should be noted that the exporter / fish company will purchase product if and if only export quality / high value product fish available. 
International Journal of Managing Value and Supply Chains (IJMVSC) Vol. 7, No. 2, June 2016

\section{Middlemen Value Chain - Fresh fish}

Table 7 shows that middlemen are getting fish either from seashore or fish villages at the average price of Rs.57.18/kg. The middlemen are investing their money on transportation charges, market fee etc. There is $38 \%$ value increase in fresh fish middlemen value chain.

Table 7. Middlemen Value Chain - Fresh fish

\begin{tabular}{|l|c|c|}
\hline Particulars of Item & $\begin{array}{l}\text { Values in } \\
\text { Rs. / Kg }\end{array}$ & $\begin{array}{l}\text { Values } \\
\%\end{array}$ \\
\hline Final Avg. sale price fixed by fishermen * & \multicolumn{2}{|c|}{57.18} \\
\hline Value Addition Operations by Middleman & & \\
\hline Avg.Sale Price fixed by Fisherman & 57.18 & $61 \%$ \\
\hline Middleman Commission & 16.5 & $18 \%$ \\
\hline Transportation Chagres & 4.36 & $5 \%$ \\
\hline Market Fee & 1.55 & $2 \%$ \\
\hline Wholesale Margin (pricing strategy) ** & 5.6 & $6 \%$ \\
\hline Retailer Margin (pricing strategy)** & 8.45 & $9 \%$ \\
\hline Final Avg.Price for Consumer & & \multicolumn{2}{|c|}{93.64} \\
\hline $\begin{array}{l}\text { PROFIT Value } \\
\text { ( Final Sale Price - Seashore Price ) }\end{array}$ & 36.46 & $38 \%$ \\
\hline $\begin{array}{l}* \text { - Avg. Sale Price Fixed by Fishermen at Seashore / Village. } \\
\text { **-The middlemen include ideal wholesale / retail margin in his price. }\end{array}$ \\
\hline
\end{tabular}

\section{HeAd loAder VAlUe Chain - FRESH FiSH}

Head loader is like door-to-door sales woman present in urban / town households and sells fish by carrying the product on her head. Table 8 shows that there is $62 \%$ price after value addition processes. The major cost goes to transportation. Return will be high because of reduced customer cost as the product is available at door step.

Table 8 : Head Loader Value Chain - Fresh Fish

\begin{tabular}{|l|c|c|}
\hline Particulars of Item & $\begin{array}{l}\text { Values in } \\
\text { Rs. / Kg }\end{array}$ & $\begin{array}{l}\text { Values in } \\
\%\end{array}$ \\
\hline Final Avg. sale price fixed by fishermen * & \multicolumn{2}{|c|}{57.18} \\
\hline Value Addition Operations by Head loader & 57.18 & $67 \%$ \\
\hline Avg.Sale Price fixed by Fisherman & 20.00 & $23 \%$ \\
\hline Cost of Transportation & 8.45 & $10 \%$ \\
\hline Margin of Head loader & \multicolumn{2}{|c|}{85.63} \\
\hline Final Avg.Price for Consumer & 28.45 & $62 \%$ \\
\hline $\begin{array}{l}\text { PROFIT Value addition } \\
\text { ( Final Sale Price - Seashore Price ) }\end{array}$ & 2 \\
\hline *- Avg. Sale Price Fixed by Fishermen at Seashore / Village. \\
\hline
\end{tabular}

\section{Cycle Carrier VAlue Chain - Fresh fish}

Cycle Carrier is like door-to-door salesman present in urban / town households and sells fish by carrying the product on a bicycle. Table 9 shows that there is $66 \%$ price after value addition processes. The major cost goes to transportation. Return will be high because of reduced customer cost as the product is available at door step. 
International Journal of Managing Value and Supply Chains (IJMVSC) Vol. 7, No. 2, June 2016

Table 9. Cycle Carrier Value Chain - Fresh Fish

\begin{tabular}{|l|c|c|}
\hline Particulars of Item & $\begin{array}{l}\text { Values in } \\
\text { Rs. / Kg }\end{array}$ & $\begin{array}{l}\text { Values in } \\
\%\end{array}$ \\
\hline Final Avg. sale price fixed by fishermen * & \multicolumn{2}{|c|}{57.18} \\
\hline Value Addition Operations by Cycle Carrier & 57.18 & $60 \%$ \\
\hline Avg.Sale Price fixed by Fisherman & 30.00 & $31 \%$ \\
\hline Cost of Transportation & 8.45 & $9 \%$ \\
\hline Margin of Cycle Carrier & \multicolumn{2}{|c|}{70.36} \\
\hline Final Avg.Price for Consumer & 13.18 & $66 \%$ \\
\hline $\begin{array}{l}\text { PROFIT Value } \\
\text { ( Final Sale Price - Seashore Price ) }\end{array}$ & \multicolumn{2}{|c|}{} \\
\hline
\end{tabular}

\section{RETAILER VALUE CHAIN - FRESH FISH}

Table 10. Retailer Carrier Value Chain - Fresh Fish

\begin{tabular}{|l|c|c|}
\hline Particulars of Item & $\begin{array}{l}\text { Values in } \\
\text { Rs. / Kg }\end{array}$ & $\begin{array}{l}\text { Values in } \\
\%\end{array}$ \\
\hline Final Avg. sale price fixed by fishermen* & \multicolumn{2}{|c|}{57.18} \\
\hline Value Addition Operations by Retailer & 57.18 & $74 \%$ \\
\hline Avg.Sale Price fixed by Fisherman & 4.36 & $6 \%$ \\
\hline Transportation Chagres & 1.55 & $2 \%$ \\
\hline Market Fee & 0.79 & $1 \%$ \\
\hline Weighing & 3.89 & $5 \%$ \\
\hline Cutting & 0.89 & $1 \%$ \\
\hline Washing & 0.45 & $1 \%$ \\
\hline Packing & 8.45 & $11 \%$ \\
\hline Retailer Margin & & 77.56 \\
\hline Final Avg.Price for Consumer & 20.38 & $59 \%$ \\
\hline $\begin{array}{l}\text { PROFIT Value } \\
\text { ( Final Sale Price - Seashore Price ) }\end{array}$ & \\
\hline *- Avg. Sale Price Fixed by Fishermen at Seashore / Village. \\
\hline
\end{tabular}

Table 10 shows that retailer will be involving in different value chain operations like cutting, washing etc. there is $59 \%$ price increase after retail value added operations to the product. 
International Journal of Managing Value and Supply Chains (IJMVSC) Vol. 7, No. 2, June 2016

\section{COMPARISON BETWEEN VALUE ADDED SELLING AND NON-VALUE ADDED SELLING}

Table 11: Comparison between value added selling and non-value added selling

\begin{tabular}{|c|c|c|c|c|c|c|}
\hline \multirow{2}{*}{$\begin{array}{c}\text { Value } \\
\text { Chain Actor }\end{array}$} & \multicolumn{2}{|c|}{ Value Added Selling } & \multicolumn{2}{c|}{$\begin{array}{c}\text { Non-Value Added } \\
\text { Selling }\end{array}$} & \multicolumn{2}{c|}{ Difference } \\
\cline { 2 - 7 } & \multicolumn{2}{|c|}{ Profit } & \multicolumn{2}{c|}{ Profit } & diff. & diff. \\
\cline { 2 - 7 } & Rs./Kg & $\%$ & Rs./Kg & $\%$ & Rs./Kg & $\%$ \\
\hline $\begin{array}{c}\text { Fishermen } \\
\begin{array}{c}\text { Intermediary } \\
\text { Marketing }\end{array}\end{array}$ & 22.18 & $38 \%$ & 7 & $17 \%$ & 15.18 & $68 \%$ \\
\hline $\begin{array}{c}\text { Fishermen } \\
\text { Direct Marketing } \\
\text { Consumer }\end{array}$ & 39.28 & $52 \%$ & 13 & $27 \%$ & 26.28 & $67 \%$ \\
\hline $\begin{array}{c}\text { Fishermen } \\
\text { Direct Marketing } \\
\text { Company }\end{array}$ & 52.18 & $59 \%$ & 25 & $31 \%$ & 27.18 & $52 \%$ \\
\hline Middlemen & 36.46 & $38 \%$ & 13 & $24 \%$ & 23.46 & $64 \%$ \\
\hline Head loader & 53.72 & $62 \%$ & 20 & $8 \%$ & 33.72 & $63 \%$ \\
\hline Cycle Carrier & 63.72 & $66 \%$ & 25 & $11 \%$ & 38.72 & $61 \%$ \\
\hline Retailer & 45.65 & $59 \%$ & 10 & $15 \%$ & 35.65 & $78 \%$ \\
\hline
\end{tabular}

The findings of the study are summarised in the Table 11. As it shows, the value chain actor and their profit after selling the product to the customer are presented.

- The fishermen practicing intermediary marketing and performing value added operations are getting $68 \%$ more returns than non-value added selling.

- The fishermen practicing direct marketing to consumer and performing value added operations are getting $67 \%$ more returns than non-value added selling.

- The fishermen practicing direct marketing to company and performing value added operations are getting 52\% more returns than non-value added selling.

- The middlemen performing value added operations are getting $64 \%$ more returns than non-value added selling.

- The middlemen performing value added operations are getting $63 \%$ more returns than non-value added selling.

- The head loader performing value added operations are getting 63\% more returns than non-value added selling.

- The cycle carriers performing value added operations are getting $61 \%$ more returns than non-value added selling.

- The retailers performing value added operations are getting $78 \%$ more returns than nonvalue added selling.

\section{CONCLUSION}

It can be concluded that in the sample of fishermen, 16 percent fishermen are not performing any value addition activities and straight away selling fish catch to either middlemen or retailer whereas 84 percent fishermen are performing value added operations ranging from two or three to six or seven different value addition processes. The most used value added activities for fresh fish are cleaning, grading, separating, washing, weighing, icing and packaging. The value addition operations by different people like fishermen, middlemen, retailer, head loader and cycle carrier 
are analysed and it is found that on an average 12 percent value is being added to fish if they perform value addition processes. Therefore it is strongly recommended to all the players of the fish supply chain to practice value chain management in order to get more value in terms of price to vendor and satisfaction to customer in terms of convenience of using the product.

\section{REFERENCES}

[1] Brown, L (1997), Competitive Marketing Strategy [M]. Melbourne: Nelson Press.

[2] Ching Chyi Lee and Jie Yang (2000), "Knowledge Value chain", Journal of Management Development, Vol.19, No.9, p.783-793.

[3] Gabriel, E. (2006), "Value Chain for Services - A new dimension of Porter's Value Chain". The IMS International Journal, Vol.34, p.1-30.

[4] Ilyas, R.M., Ravi Shankar., Banwet, D.K., (2008b), "Creating flex-lean-agile value chain by outsourcing - An ISM based interventional roadmap", Business Process Management Journal, Vol. 14, No. 3, p.338-389.

[5] Kaplinsky, R. and M. Morris (2001), “A Handbook for Value Chain Research”, Prepared for the International Development Research Centre (IDRC), p.4-6.

[6] Lynch, R. (2003), Corporate Strategy, 3rd ed., Prentice Hall Financial Times.

[7] Pathania-Jain, G. (2001), "Global parents, local partners: A value-chain analysis of collaborative strategies of media firms in India", Journal of Media Economics, Vol. 14, No. 3, p. 169-187.

[8] Porter, M. E. (1990), The competitive advantage of nations, New York: Free Press.

[9] Porter M. E (1985), Competitive Advantage: Creating and Sustaining Superior Performance, New York: The Free Press.

[10] Rejean Landry, Nabil Amara, Ariel Pablos-Mendeo, Ramesh Shademani \& Irving Gold (2006), "The knowledge-value chain: a conceptual framework for knowledge translation in health", Bulletin of the World Health Organization, Vol.84, No.8, p.597-602.

[11] Seyed Mohammad Adeli, Silvia Elaluf Calderwood, Claus Oskar Heintzeler, Javier Huerta and Caroline Legler (2012), "Mobile Value Chain and Services", MOBILITY: The Second International Conference on Mobile Services, Resources and Users.

[12] SustainAbility, UNEP and UNGC (2008), Unchaining Value: Innovative approaches to sustainable supply, p.2.

[13] Van der Merwe, A. \& Cronje, J. (2004), "The educational value chain as a modelling tool in reengineering efforts", Proceedings of the 2004 international Symposium on information and Communication Technologies, ACM International Conference Proceedings Series, Vol.90, No.1, p.122-127.

[14] Vorster, A. (2001), "Planning for value in the mining value chain", The Journal of The South African Institute of Mining and Metallurgy, March/April, p.61-68. Wang Aimin \& Li Shunxi (2011), “A Model of Value Chain Management Based on Customer Relationship Management", Journal on Innovation and Sustainability, Vol.2, No.3, p.17-21.

[15] Walters D., Lancaster G (2000), Implementing Value Strategy through the Value Chain, Management Decision, 38(3), pp 160-178.

[16] WBCSD (2011), Collaboration, innovation, transformation: Ideas and inspiration to accelerate sustainable growth - A value chain approach, p. $3 \& 5$.

\section{Author}

Mr.V.V.Devi Prasad Kotni is working as Assistant Professor from the last eight years in Gayatri Vidya Parishad College for Degree and PG Courses (Autonomous), Visakhapatnam, Andhra Pradesh. He also has Industry experience of four years. He published about 45 research papers (19 papers in International Journals, 11 papers in National Journals and 15 papers in Seminar proceedings) and two books into his account. He presented 23 papers in International / National Seminars and conferences. He presented papers in Bangkok and Kaulalumpur (sponsored by AICTE) and another

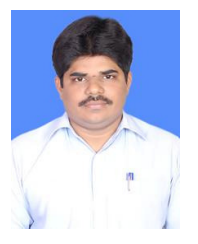
paper in New York (sponsored by UGC - Travel Grant). He completed one UGC major research project in 2012 as co-investigator and one UGC Minor Research Project in 2016 as principal investigator. He was awarded AICTE - Career Award for Young Teacher research grant in 2013. Also He completed two consultancy projects as project leader. He is serving as editorial board member for four journals. 